\title{
Modelling tumour volume variations in head and neck cancer: contribution of magnetic resonance imaging for patients undergoing induction chemotherapy
}

Modificazioni del volume nei tumori della testa e del collo: il contributo delle immagini di risonanza magnetica nei pazienti sottoposti a chemioterapia di induzione

\begin{abstract}
N. DINAPOLI ${ }^{1}$, T. TARTAGLIONE${ }^{2}$, F. BUSSU ${ }^{3}$, R. AUTORINO ${ }^{1}$, F. MICCICHË ${ }^{1}$, M. SCIANDRA², E. VISCONTI², C. COLOSIMO², G. PALUDETTI ${ }^{3}$, V. VALENTINI ${ }^{1}$

${ }^{1}$ Institute of Radiotherapy, Università Cattolica del Sacro Cuore, Rome, Italy; ${ }^{2}$ Institute of Radiology, Università Cattolica del Sacro Cuore, Rome, Italy; ${ }^{3}$ Institute of Otolaryngology, Università Cattolica del Sacro Cuore, Rome, Italy
\end{abstract}

\section{SUMMARY}

Primary tumour volume evaluation has predictive value for estimating survival outcomes. Using volumetric data acquired by MRI in patients undergoing induction chemotherapy $(I C)$ these outcomes were estimated before the radiotherapy course in head and neck cancer (HNC) patients. MRI performed before and after IC in 36 locally advanced HNC patients were analysed to measure primary tumour volume. The two volumes were correlated using the linear-log ratio $(L L R)$ between the volume in the first MRI and the volume in the second. Cox's proportional hazards models (CPHM) were defined for loco-regional control (LRC), disease-free survival (DFS) and overall survival $(O S)$. Strict evaluation of the influence of volume delineation uncertainties on prediction of final outcomes has been defined. LLR showed good predictive value for all survival outcomes in CPHM. Predictive models for LRC and DFS at 24 months showed optimal discrimination and prediction capability. Evaluation of primary tumour volume variations in HNC after IC provides an example of modelling that can be easily used even for other adaptive treatment approaches. A complete assessment of uncertainties in covariates required for running models is a prerequisite to create reliable clinically models.

KEY WORDS: Magnetic resonance imaging • Head and neck cancer • Induction chemotherapy • Survival modelling

\section{RIASSUNTO}

La valutazione del volume del tumore primitivo ha mostrato un valore predittivo per la stima dei risultati della sopravvivenza. Usando $i$ dati volumetrici acquisiti con la Risonanza Magnetica (RM) nei pazienti sottoposti a chemioterapia di induzione (CI), tali risultati sono stati stimati nei pazienti con tumore del testa e collo, prima del trattamento radiante. Le immagini RM acquisite prima e dopo CI in 36 pazienti con tumore avanzato della testa e del collo sono state analizzate per valutarne il volume del tumore primitivo. I due volumi sono stati correlati utilizzando la regressione lineare locale tra $i$ volume valutati nelle immagini della prima e quelli della seconda RM. Sono stati definiti i modelli di rischio proporzionale di COX per la valutazione del controllo locoregionale, la sopravvivenza libera da malattia e la sopravvivenza globale. La regressione lineare locale ha mostrato un buon valore predittivo per tutti i risultati di sopravvivenza nei modelli di rischio proporzionale di COX. I modelli predittivi per il controllo locoregionale di malattia e la sopravvivenza libera da malattia a 24 mesi ha mostrato una ottima discriminazione e capacità di previsione. La valutazione delle variazioni dei volumi dei tumori primitivi della testa e del collo dopo CI fornisce un esempio di modello che può essere facilmente utilizzato per altri approcci terapeutici. Una valutazione completa delle variabili nelle covariate è un prerequisito necessario per la creazione di modelli clinicamente attendibili.

PAROLE CHIAVE: Risonanza magnetica $\bullet$ Tumori della testa e del collo $\bullet$ Chemioterapia di induzione $\bullet$ Modello di sopravvivenza

Acta Otorhinolaryngol Ital 2017;37:9-16

\section{Introduction}

Treatment of head-and-neck cancer (HNC) now requires complex multi-specialistic management and HNC types are typically characterised by wide heterogeneity of anatomical sites, primary treatment approaches and diagnostic pitfalls, the latter increasingly connected with procedures of optimisation of radiotherapy treatment planning ${ }^{1-4}$. Treatment of $\mathrm{HNC}$ is mainly based on integrating a chemoradiation approach ${ }^{5-7}$, and in this context the use of induction chemotherapy (IC) has shown a consistent number of clinical responses before the start of radiotherapy ${ }^{8}$. The role of this approach is still under debate because of unclear advantages in final survival outcomes after definitive radiochemotherapy (RTCT) compared to 
treatment based only on concurrent RTCT $^{9}$. The individualisation of cancer treatment is an increasingly important topic in the medical literature ${ }^{10}$. Starting from this background, the use of IC provides the chance to analyse changes in tumour appearance, where imaging techniques can be evaluated moving towards adaptive radiotherapy strategies ${ }^{11}$. Traditional tumour evaluation approaches, such as tumour volume delineation on simulation computed tomography, have already proven to be consistent in terms of outcome correlations ${ }^{12}$. The aim of this study was to evaluate the feasibility and potential outcome classification for HNC patients undergoing IC, by using MRI based primary tumour volume shrinking evaluation after IC. After modelling, survival outcomes on imaging data a nomogram followed by strict model evaluation are assessed. This evaluation procedure is the main goal of this paper, being the base of further evaluations that can be extended to other adaptive treatment procedures.

\section{Materials and methods}

\section{Patient characteristics}

Thirty-six locally-advanced HNC patients were retrospectively analysed in our institution by selecting cases that underwent IC before the administration of definitive RTCT (34 patients) or before undergoing surgery (2 patients with oral cavity cancer, these latter underwent adjuvant RTCT after surgery). All patients gave informed consent for inclusion in the study. Main selection criteria were availability of complete on-site MRI diagnostics in patients with locally advanced HNC (stage III or IV, without distant metastases). Patient staging, chemotherapy and radiotherapy details are summarised in Table I. All patients were evaluated using MRI before and after IC. After RTCT patients were evaluated by MRI at $1^{\text {st }}$ followup time to assess response to treatment. Subsequently, follow-up was routinely performed using CT, MRI and PET-CT to detect loco-regional or distant failures.

\section{Treatment details}

IC was administered using a TPF chemotherapy regimen: taxotere $75 \mathrm{mg} / \mathrm{m}^{2}$ (day 1), cisplatin $75 \mathrm{mg} / \mathrm{m}^{2}$ (day 1) and 5-flurouracil $750 \mathrm{mg} / \mathrm{m}^{2}$ in continuous infusion (days 1-4) every three weeks for 3 cycles. Radiotherapy was delivered using a linear accelerator, and the dose and technique are summarised in Table 1. Lymphatic target volumes for radiation treatment were delineated according to Gregoire's indications for neck-positive and post-operative necks ${ }^{13}$. Concurrent cisplatin based chemotherapy (2 cycles at $100 \mathrm{mg} /$ $\mathrm{m}^{2}$ at the beginning of radiotherapy and after 3 weeks) was administered in 35 patients, while 1 patient received concurrent cetuximab at $250 \mathrm{mg} / \mathrm{m}^{2}$. The outcomes evaluated were loco-regional control (LRC), disease-free survival (DFS) and overall survival (OS).
Table I. Summary of patient characteristics and RTCT treatment.

\begin{tabular}{|c|c|c|c|}
\hline Patient characteristics & & Number & $(\%)$ \\
\hline \multirow[t]{6}{*}{ Primary tumour site } & Oropharynx & 15 & $(41.7)$ \\
\hline & Nasopharynx & 14 & $(38.9)$ \\
\hline & Larynx & 3 & $(8.3)$ \\
\hline & Oral cavity & 2 & (5.5) \\
\hline & Hypopharynx & 1 & $(2.8)$ \\
\hline & Nasal cavity & 1 & $(2.8)$ \\
\hline \multirow[t]{3}{*}{ Stage } & $\mathrm{IV}^{*}$ & 34 & $(94.4)$ \\
\hline & III & 2 & (5.6) \\
\hline & Dose [Gy] & & \\
\hline \multirow{4}{*}{$\begin{array}{l}\text { PTV1 } \\
\text { (primary + margin) }\end{array}$} & 70.2 & 33 & (91.6) \\
\hline & 68.4 & 1 & $(2.8)$ \\
\hline & 64.8 & 1 & $(2.8)$ \\
\hline & $50.4 \dagger$ & 1 & $(2.8)$ \\
\hline \multirow{5}{*}{$\begin{array}{l}\text { PTV2 } \\
\text { (positive lymphatic } \\
\text { compartment) }\end{array}$} & 64.8 & 15 & $(41.6)$ \\
\hline & 61.2 & 2 & $(5.6)$ \\
\hline & 59.4 & 16 & $(44.4)$ \\
\hline & $50.4 \dagger$ & 2 & (5.6) \\
\hline & 36 & 1 & (2.8) \\
\hline \multirow{5}{*}{$\begin{array}{l}\text { PTV3 } \\
\text { (elective lymph nodes) }\end{array}$} & 64.8 & 2 & $(5.6)$ \\
\hline & 59.4 & 23 & $(63.8)$ \\
\hline & 50.4 & 9 & $(25)$ \\
\hline & 36 & 1 & $(2.8)$ \\
\hline & 30.6 & 1 & $(2.8)$ \\
\hline \multirow[t]{2}{*}{ RT technique } & IMRT & 35 & $(97.2)$ \\
\hline & 3D CRT & 1 & $(2.8)$ \\
\hline \multirow[t]{2}{*}{ Concomitant chemotherapy } & CDDP & 35 & (97.2) \\
\hline & Cetuximab & 1 & $(2.8)$ \\
\hline
\end{tabular}

${ }^{*}$ No distant metastases at diagnosis.

t One of the two post-operative patients was treated using a two volumes approach in CTV delineation, with maximum delivered dose 50.4 Gy. All treatments were delivered at 1.8 Gy per fraction.

\section{Magnetic resonance evaluation}

Volumetric evaluation of primary tumours was performed using OsiriX Imaging Software (http://www.osirix-viewer.com): contours of primary lesions were manually outlined by two different radiologists who are expert in HNC imaging, in order to evaluate the impact of inter-observer differences in delineation. Volumes were delineated on axial $\mathrm{T} 2 \mathrm{w}$ images, also using other sequences, mostly T1w images, before and after intravenous contrast injection, to better refine the delineation (Fig. 1). When both radiologists were satisfied with the outlines, tumour volumes were finally calculated.

\section{Statistical analysis}

Statistical analysis was performed using R Statistical Software (R Core Team 2013. http://www.R-project. org/). All statistical tests considered an alpha level of $5 \%$ to indicate statistical significance. The series of volumes delineated by each radiologist (labelled ' $a$ ' and ' $b$ ') be- 


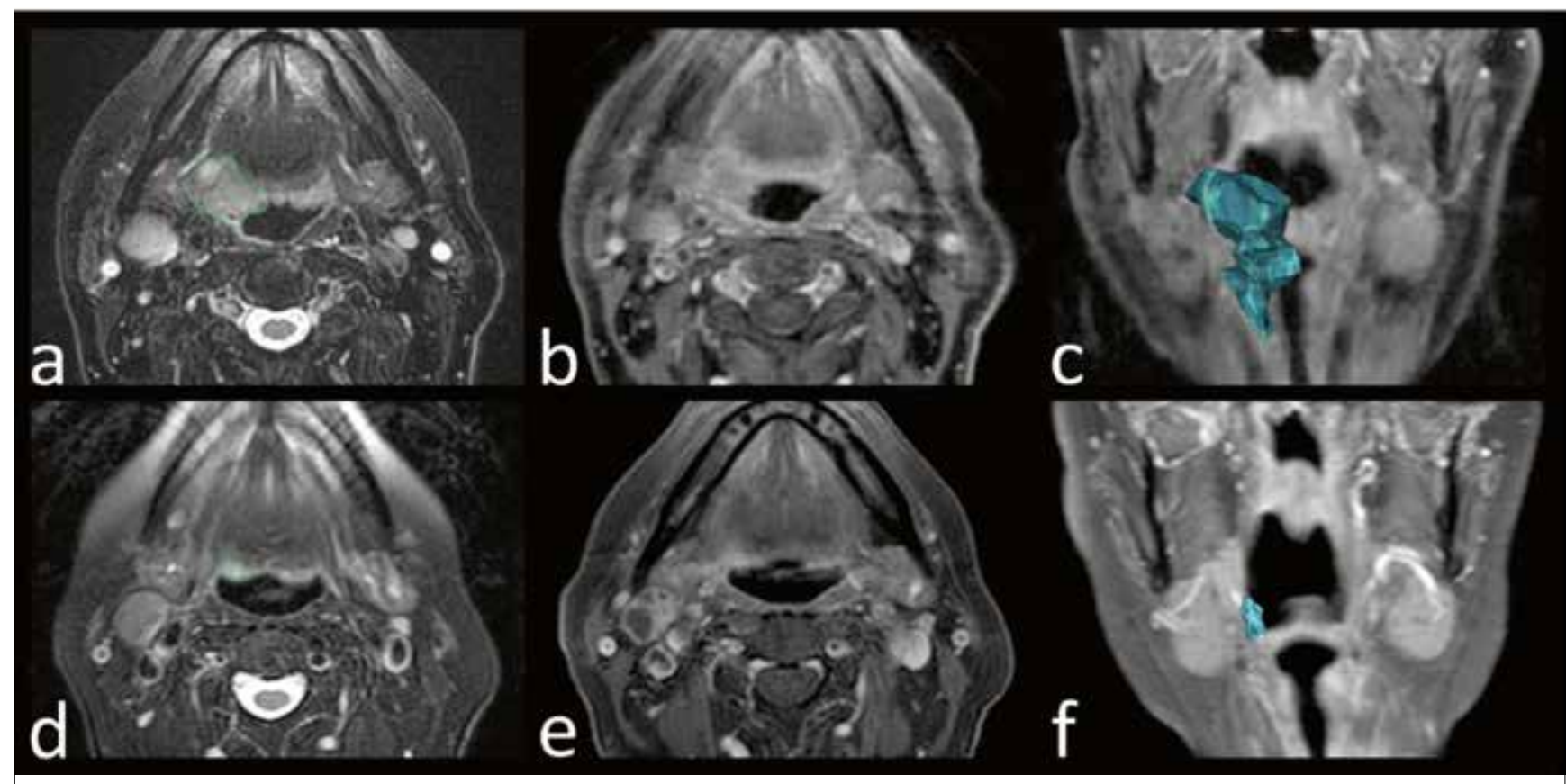

Fig. 1. Volumetric assessment of primary tumour volume. Squamous cell carcinoma of the oropharynx in a 73-year-old man. (a,d) Axial fat-saturated T2-weighted MR images. $(b, e)$ Axial post-contrast T1-weighted fat-saturated MR images. (c,f) Coronal post-contrast T1-weighted fat-saturated MR images with 3D volumetric tumour reconstructions. Before induction chemotherapy (a,b,c): MR images show expansive/infiltrative tissue centred on the right glossopharyngeal fold, hyper-intense on T2weighted image (a) with slight and faint enhancement on post-contrast T1-weighted fat-saturated MR image (b). The lesion was manually outlined (green closed line in a) to obtain a volumetric reconstruction of the tumour (blue volume in c). After induction chemotherapy (d,e,f) MR images show significant volumetric reduction of the lesion.

fore $\left(V_{1} a, V_{1} b\right)$ and after $\left(V_{2} a, V_{2} b\right)$ IC were analysed to detect normal distribution of values. Correlation between volumes measured at each step by each radiologist $\left(V_{1} a\right.$ vs. $V_{1} b, V_{2} a$ vs $\left.V_{2} b\right)$ was calculated to assess homogeneity in delineation between the operators. Afterwards, two series of mean values of volumes for each diagnostic step

were calculated $\left(m V_{1}=\frac{V_{1} a+V_{1} b}{2}, m V_{2}=\frac{V_{2} a+V_{2} b}{2}\right)$,

and used to divide patients in subsets to calculate stratified log-rank tests for survival outcomes. To define the relationship between volumes in the two diagnostic steps, two regression models were calculated, the first using linear regression between $m V_{2}$ over $m V_{1}$, and the second between $m V_{2}$ over $\log \left(m V_{1}\right)$. Considering the higher predictive power of the log-linear regression in describing the relationship between $m V_{2}$ and $m V_{1}$ (see results) a new score was calculated (Linear-Log-Ratio, $L L R)$ :

$$
L L R=\frac{m V_{2}}{\log \left(m V_{1}\right)}
$$

and used in Cox's proportional hazards model (CPHM) regression together with the values of $m V_{1}$ and $m V_{2}$ to find the best significant predictor for survival outcomes. Backward elimination process was used for determining the entry and removal of variables from the models with significance levels, respectively, of 0.05 and 0.15 . After calculation of CPHMs, we tested the discrimination ability of the models using Harrell's c-index ${ }^{14}$ calculated by bootstrap analysis on 1000 of a randomly created dataset based on the first. The prediction power of the models was defined using calibration plots drawn by resampling 200 cases for each model and calculating the mean prediction error as difference between observed - predicted outcome in the series ${ }^{14}$. Considering the problem of inter-subject (but also intra-subject) variations in volume delineation that arises from the literature in many anatomical sites and using different delineation procedures or imaging modalities ${ }^{15-24}$, a procedure to evaluate the susceptibility of the model to variations in the delineation was defined: we created a function of $m V_{1}$ and $m V_{2}$ that gives the value of punctual uncertainty using the concept of 'gradient' of a function in two variables. The chosen function for the analysis was DFS at 24 months, achieved by CPHM. Considering the appearance of the survival function at 24 months in a three-dimensional coordinates space (Fig. 2, DFS at 24 months) as a function of two variables $\left(m V_{1}\right.$ and $\left.m V_{2}\right)$ linked by LLR, we can easily find portions of the surface where the slope of the function is higher, corresponding to values of survival prediction with high susceptibility to variability in volume delineation. In order to provide a quantification of the value of this susceptibility the gradient of survival function was 




Fig. 2. Plot of $3 D$ surface showing DFS at 24 months as a function of mean tumour volume before $\left(m V_{1}\right)$ and after $\left(m V_{2}\right)$ induction chemotherapy: the slope of the surface can vary according to the values of the two volumes, being steeper in the left corner of the plot, where the values of $m V_{1}$ and $m V_{2}$ are close to zero.

calculated using $m V_{1}$ and $m V_{2}$ as parameters of the function. Being:

$$
S\left(m V_{1}, m V_{2}\right)=\exp \left\{-H_{0} t \cdot \exp \left[\beta \cdot m V_{2} / \log \left(m V_{1}\right)\right]\right\}
$$

the value of survival function at time $t$ (it can be used either for LRC or DFS) with $H_{0} t$ the baseline hazard at chosen time (in our case 24 months), _ the coefficient of the covariate calculated with CPHM (see Table II), we first calculated the partial derivatives of $S$ with respect to $m V_{1}$ and $m V_{2}$ and gave a first order estimate of the variation of $S$ as a function of $m V_{1}$ and $m V_{2}$ :

$$
\Delta S\left(m V_{1}, m V_{2}\right) \cong\left|\left(\frac{\partial S}{\partial m V_{1}}, \frac{\partial S}{\partial m V_{2}}\right)\right|=\sqrt{\left(\frac{\partial S}{\partial m V_{1}}\right)^{2}+\left(\frac{\partial S}{\partial m V_{2}}\right)^{2}}
$$

The result of this equation is function of $m V_{1}$ and $m V_{2}$ only, and can be used to assess the sensitivity to error in volume delineation: it corresponds to the punctual maximum variation that survival function can show by chang- ing the values of $m V_{1}$ and $m V_{2}$; looking at the 3D graph of survival function it is the module of the vector in one point, tangent to the direction of the highest steepness of the surface in such point. Using this method it is possible to determine both the predicted survival result and the level of uncertainty that matches the prediction given by the model. This last analysis was performed using Wolfram Mathematica ${ }^{\circledR}$ 9.0.1.0 (@1988-2013 WolframResearch Inc. Champaign, IL). Finally, a nomogram for DFS to directly calculate the predicted outcome using the two measures of MRI tumour volume, before and after IC ( $m V_{1}$ and $\left.m V_{2}\right)$, was created. It was delineated using Pynomo, a program to create nomograms using Python interpreter (http://www.pynomo.org).

\section{Results}

The two volume series delineated by each radiologist (labelled ' $a$ ' and 'b') before $\left(V_{1} a, V_{1} b\right)$ and after $\left(V_{2} a, V_{2} b\right)$ IC analysed using D'Agostino-Pearson test showed non- 
Table II. Summary of Cox's proportional hazards models for the LLR (Linear-Log-Ratio) covariate. All models and single LLR covariates in each model are largely significant (P-Values < 0.05 in all cases). The bootstrap over 1000 resampled series for each model allowed to calculate Harrell's c-index decreased by the 'optimism' for preventing model overfitting in starting case series. The performance of models is very close to the original c-index in all cases, meaning high discriminating power. The evaluation of the mean error of prediction of survival outcomes at 24 months, through calibration on 200 resampled cases, is also provided.

\begin{tabular}{|c|c|c|c|c|c|c|c|c|}
\hline \multicolumn{9}{|c|}{ Summary of Cox's Proportional Hazards Regression models with unique significant covariate } \\
\hline Outcome & $\begin{array}{l}\text { Model P-value } \\
\text { (Likelihood ratio } \\
\text { test) }\end{array}$ & $\begin{array}{l}\text { Standard } \\
\text { error }\end{array}$ & $\begin{array}{l}\text { P-value } \\
\operatorname{Pr}(>|z|)\end{array}$ & $\begin{array}{l}\text { Standard } \\
\text { error }\end{array}$ & $\begin{array}{l}\text { Harrell's } \\
\text { c-index (c) }\end{array}$ & Optimism (0p) & $\begin{array}{l}\text { Corrected } \\
\text { Harrell's } \\
\text { c-index } \\
(c-0 p / 2)\end{array}$ & $\begin{array}{c}\text { Mean calibration } \\
\text { error } \\
\text { (24 months } \\
\text { prediction) }\end{array}$ \\
\hline LRC & 0.0013060 & 0.4271 & 0.000788 & 0.1272 & 0.7668 & 0.0105 & 0.7615 & 0.073 \\
\hline DFS & 0.0006376 & 0.3427 & 0.000234 & 0.0931 & 0.7546 & 0.0034 & 0.7529 & 0.056 \\
\hline OS & 0.0008928 & 0.4905 & 0.000771 & 0.1458 & 0.8000 & 0.0155 & 0.7923 & 0.062 \\
\hline
\end{tabular}

normal distribution (in all four series $\mathrm{P}<0.0001$ ). Thus, non-parametric tests were used to assess correlation between $V_{1} a$ vs $V_{1} b$ and $V_{2} a$ vs $V_{2} b$ (Spearman's coefficient of rank correlation and Kendall's Tau $\mathrm{P}<0.0001$ in both cases). These tests showed an optimal consistence in contouring procedure, and were not biased by different operators. All subsequent statistical analyses were carried out using the mean value of volume measures before $\left(m V_{1},\right)$ and after $\left(m V_{2}\right)$ IC. Each series of mean values was used to determine factors for performing stratified Kaplan Meier (KM) analysis for LRC, DFS and OS: using the median value of $m V_{1}$ and $m V_{2}$ as cut-off (thus dividing the population into equal subsets, each one with 18 patients).

The KM Logrank test for $m V_{1}$ (cut-off: median $=12.9744 \mathrm{cc}$, range: 2.209 to $96.7385 \mathrm{cc}$ ) was not significant in all tested outcomes, while the same test for $m V_{2}$ (cut-off: median $=4.2181 \mathrm{cc}$, range: 0 to $39.3675 \mathrm{cc}$ ) was always significant (LRC: P-value $=0.0003$, $\mathrm{HR}=8.9437,95 \% \mathrm{CI}=2.9196$ to $27.3972 ;$ DFS: $\mathrm{P}=0.0011, \mathrm{HR}=5.9193,95 \% \mathrm{CI}=2.1952$ to 15.9613 ; OS: $\mathrm{P}=0.0097, \mathrm{HR}=5.7386,95 \% \mathrm{CI}=1.8458$ to 17.8412). In order to detect a correlation between $m V_{1}$ and $m V_{2}$ two regression functions were calculated and compared: the first. using a straight linear regression ( $\left.m V_{2}=\beta \cdot m V_{1}+\varepsilon\right)$, showed a significant overall $\mathrm{P}$ $<0.0001, b \mathrm{P}<0.0001, e \mathrm{P}=0.402$ (n.s.), adjusted $\mathrm{R}^{2}$ $=0.3796$; the second, using a regression with log-transformation of variable $m V_{1}, \quad\left(m V_{2}=\beta \cdot \log \left(m V_{1}\right)+\varepsilon\right.$ ), showed a significant overall $\mathrm{P}<0.0001, b \mathrm{P}<0.0001$ and also an $e \mathrm{P}=0.00525$ (meaning that there is a better regression fit using log transformation rather than the simple correlation shown with lacking significance in the $e$ term for the linear model) and finally a higher predictive power shown by higher adjusted $\mathrm{R}^{2}=0.4329$.

Starting from this evidence, which links the concept of tumour regression with the biological assumption of fractional killing due to chemotherapy administration ${ }^{2526}$, an assumption proven for fractionated radiotherapy ${ }^{27}$, we considered the possibility to create three different CPHM using LRC, DFS and OS as outcome, and both values of the volumes ( $m V_{1}$ and $\left.m V_{2}\right)$ together with the value of linear-log ratio (LLR) as covariates, being such covariates dependent each other from the regression. Using the process of backward elimination of covariates we found a consistent significance for $L L R$ that measures volume shrinkage against all checked survival outcomes, while $m V_{1}$ and $m V_{2}$ were no longer significant.

The results of CPHM are summarised in Table II. In order to assess the reliability of the models Harrel's c-index ${ }^{14}$ was calculated for each model. Using a bootstrapping procedure, over 1000 randomly resampled datasets, we prevented the effect of overfitting by decreasing the value of the c-index: the value of optimism as defined by Harrel, performance of fitted model compared to that expected by chance ${ }^{14}$, was always very low (Table II), showing an overall good discrimination performance of initially fitted models. The calibration plots ${ }^{28}$ of the models showed a small underestimation of the predicted outcomes with respect to the observed outcomes (Table II).

Considering as sufficient the number of patients at risk ${ }^{29}$ at 24 months of survival (16 patients), with a median follow-up time of 27 months (range 6-46), we developed a nomogram for DFS, calculating predicted survival as a direct function of $m V_{1}$ and $m V_{2}$ at 24 months. For OS the median follow-up time was considered too short to ensure a clinically reliable model, despite the significance in the modelling procedure, while LRC was considered redundant respect to the DSF evaluation in our case series. No stratification of patients according tumour primary site was performed, because of lack of significance in KaplanMeier LRC, DFS and OS according to this factor.

In Figure 3 the nomogram showing predicted DFS at 24 months is shown. The use of this nomogram does not require calculating sums, but only placing a ruler connecting the value of the $m V_{1}$ on the right with the value of the $m V_{2}$ on the left. The predicted value of DFS at 24 months can be read on the oblique line showing the outcome prediction where the connection intersects this line. An aspect usually not analysed using current predictive models evaluation and nomogram drawing procedures ${ }^{30}$ is the need to assess the level of uncertainty (that is error) in 


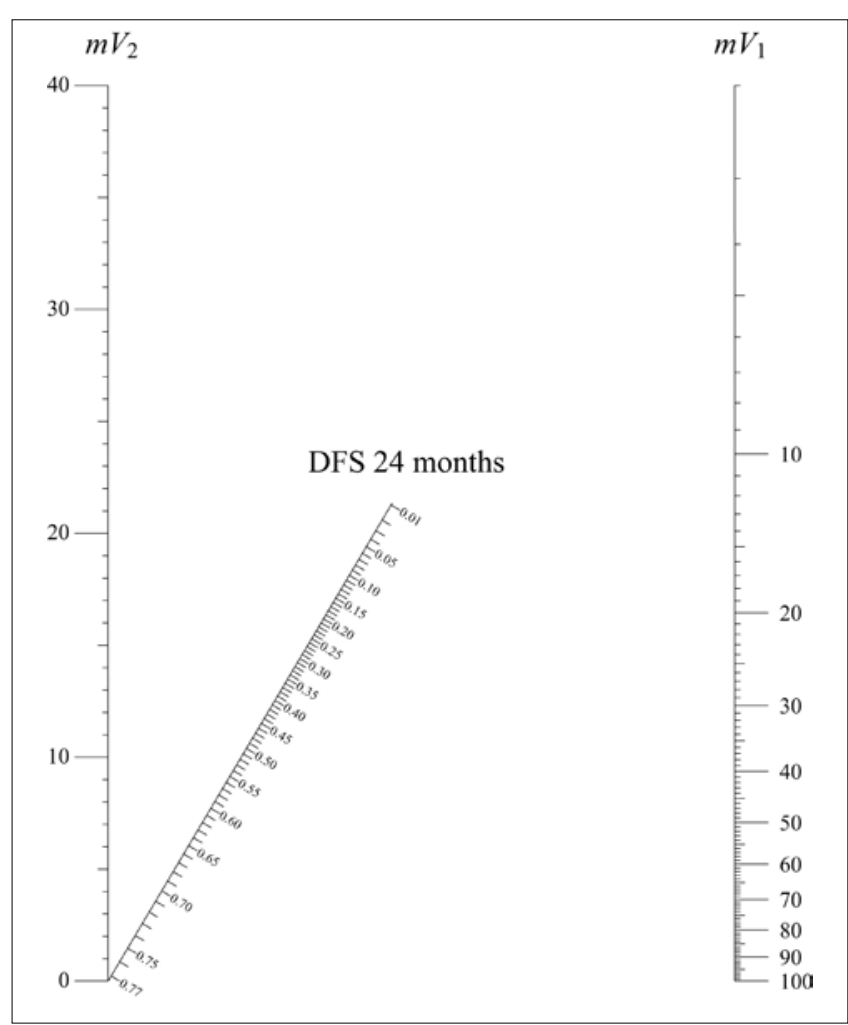

Fig. 3. Nomogram for calculating disease-free survival (DFS) at 24 months. Two vertical lines show the values of mean tumour volume before $\left(m V_{1}\right)$ and after $\left(m V_{2}\right)$ induction chemotherapy. Using a ruler to draw a straight line connecting the values of the two volumes on the oblique outcome line the predicted survival probability can be directly read.

measures of covariates put into the model. Covariates as sex, age and similar, used within predictive models, usually do not need to be evaluated for errors in detecting the value (especially when considering simple dummy variables). Indeed, when managing variables such as the value of delineated volume, as in our study, or similar measures subject to some kind of detection error, a verification of the variability in outcome prediction due to uncertainties in covariates should always be considered: as cited previously, there is much evidence for great variability in volume delineation procedures, that can vary to according anatomical site, imaging modality etc. ${ }^{15-2431}$.

This situation led us to use the procedure described in the previous section to assess the susceptibility of the model to variations in volume delineation using the gradient function derived from the survival functions calculated by CPHMs. The values of the gradient as a function of $m V_{1}$ and $m V_{2}$ for DFS are shown in Figure 4. This plot directly shows the maximum variation of survival prediction for small changes in covariates. In every point of the plot, the error in the determination can be always considered lower than or equal to the value shown by curved lines. As shown in Figure 4, only for very small values of $m V_{1}$ $(<12.5 \mathrm{cc})$ and $m V_{2}(<6 \mathrm{cc})$ did the amount of delineation error become significant in affecting the predicted outcome, a prediction that can vary more than $5 \%$ in a very small portion of the parameter space (area shown by the continuous line in the down-left corner).

\section{Discussion and conclusions}

The evaluation of response in patients undergoing to treatment for different tumour types is one of the key-points in adaptive radiotherapy (ART), but the study of geometrical or volumetric changes in tumour volume and their subsequent impact on survival outcomes are still poor.

MR and CT are non-invasive imaging techniques that have an important role in assessing response to therapy, evaluating both morphological and "functional" parameters ${ }^{32}$. These parameters, as described in the literature, can be useful tools in oncological management. In fact, it is possible to characterise tissue cellularity by evaluating the motion of water molecules on MR diffusion-weighted imaging (DWI) and to provide information regarding tumour perfusion and permeability with MR perfusion-weighted imaging (PWI) or perfusion-CT (PCT) studies. DWI is described to be a predictor of response to therapy and a good tool to differentiate between recurrent tumours from post-radiation changes ${ }^{33-34}$. PWI may play a role in detecting residual disease and predict patient outcomes ${ }^{35} 36$. PCT, as well, may be helpful to differentiate between post-therapeutic changes and tumour recurrence, to monitor patients after radiotherapy and/or chemotherapy and predict response after induction chemotherapy ${ }^{37}{ }^{38}$. In this study, we highlight the meaningful contribution of morphological MRI through the assessment of changes in tumour volume before and after CI to predict survival outcomes of HNC patients undergoing definitive RTCT. This approach can be considered an assumption to be addressed by other types of strict radiotherapy 'adaptive' approaches. Indeed, despite the relative small number of cases, the relationship between survival outcomes and changes in tumour volumes shown by this analysis is consistent in HNC patients undergoing CI.

The modelling of CPHM using a non-linear relationship between two observed volumes allows to overcome the problem of co-linearity ${ }^{39}$ when analysing parameters that are related each other, as the two volumes are. Furthermore, the use of LLR implies identification of the effect of treatment on tumour volume, as calculated by considering the possibility of tumour shrinkage rather than no change or enlargement in tumour volume. From a clinical point of view, this correlation has stronger predictive value than using the simple values of $m V_{1}$ and $m V_{2}$, because as a consequence of the assumptions of CPHM the two volumes should be independent in conditioning the outcome ${ }^{40}$, but this assumption cannot be considered feasible in this model. The possibility to obtain a consistent 'benchmark' to refer the outcome prediction in a single predictor, being the definition of the LLR based on biological and clinical assumptions, confirms the result despite the small number 


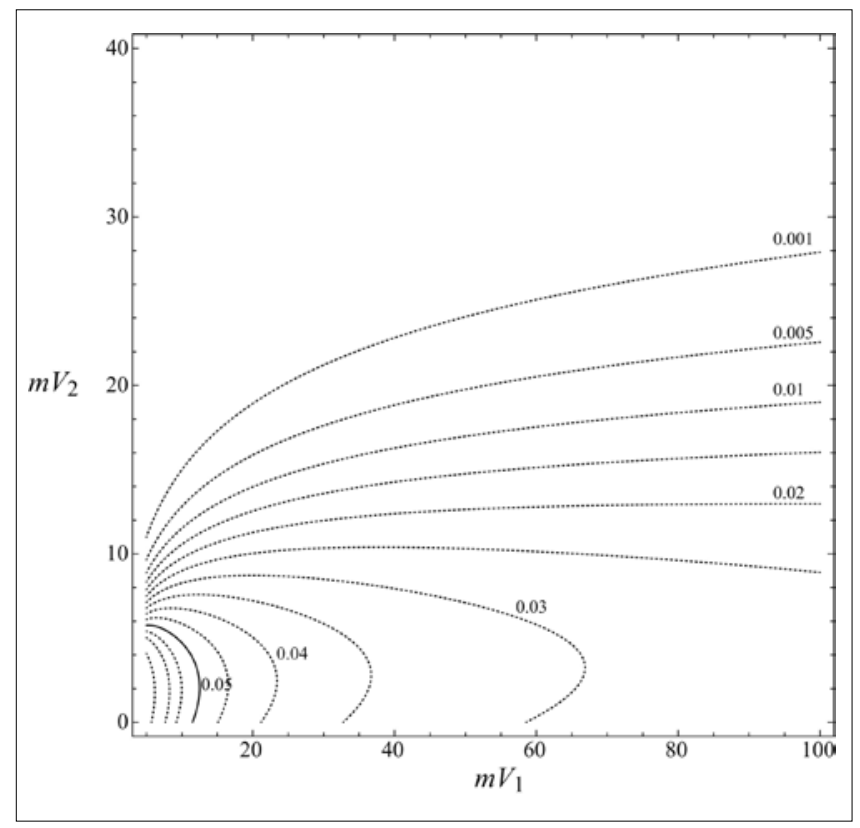

Fig. 4. Plot of gradient function. This plot shows the values of maximum variation in the outcome as a function of $m V_{1}$ and $m V_{2}$. It corresponds to maximum level of error for the survival function and can be used to assess the reliability of the prediction after survival calculation. In the down-left corner of the plot small variations of $m V_{1}$ and $m V_{2}$ can lead to outcome variations larger than $5 \%$ (continuous line). For most of the graph, the level of uncertainty can be considered reasonably low.

of cases and heterogeneity of the patient cohort. In the literature, there are already examples of correlations between survival outcomes and tumour volume assessment, but in HNC have been limited to diagnosis ${ }^{1241-43}$. In cervical cancer, there is evidence of a semi-logarithmic relation between tumour volume at diagnosis and subsequent measures achieved by weekly MRI ${ }^{44}$, and this relation was confirmed in our study for HNC after CI. As stated before, the semi-logarithmic volume reduction is the mathematical translation of the fractional killing phenomenon ${ }^{25-27}$, due to constant proportion between the number of killed and surviving tumour cells for each therapeutic event that is the single radiotherapy fraction ${ }^{44}$ or the single chemotherapy administration as in our study. The real value of the modelling procedure described in this work is not in the predictive value of the model itself, which gives only the evaluation of the results for this single case series and not yet validated by any external dataset, but rather it is in the method chosen for data analysis and evaluation of the pitfalls hidden in the volume assessment.

In this case, considering the small number of patients, better performance could be achieved by increasing the number of cases or refitting the models by introducing other clinical covariates that can improve the predictive power of the survival functions. The results of this study imply that measurement of the tumour volume can be considered a good predictor for patients undergoing CI and the strong correlation among outcomes and LLR can be an helpful parameter to be evaluated in perspective studies with 'adaptive' approaches to treatment. Hopefully, a similar approach to evaluate measures achieved by different diagnostic procedures could be used to better refine the actual impact of survival models in describing outcomes based on imaging studies.

\section{References}

1 Shah GV, Wesolowski JR, Ansari S, et al. New directions in head and neck imaging. J Surg Oncol 2008;97:644-8.

2 Daisne J-F, Duprez T, Weynand B, et al. Tumor volume in pharyngolaryngeal squamous cell carcinoma: comparison at CT, MR imaging, and FDG PET and validation with surgical specimen. Radiology 2004;233:93-100.

3 Vandecaveye V, Dirix P, De Keyzer F, et al. Predictive value of diffusion-weighted magnetic resonance imaging during chemoradiotherapy for head and neck squamous cell carcinoma. Eur Radiol 2010;20:1703-14.

4 Van Baardwijk A, Baumert BG, Bosmans G, et al. The current status of FDG-PET in tumour volume definition in radiotherapy treatment planning. Cancer Treat Rev 2006;32:245-60.

5 Baujat B, Audry H, Bourhis J, et al. Chemotherapy in locally advanced nasopharyngeal carcinoma: an individual patient data meta-analysis of eight randomized trials and $1753 \mathrm{pa}$ tients. Int J Radiat Oncol Biol Phys 2006;64:47-56.

6 Bourhis J, Le Maître A, Baujat B, et al. Individual patients' data meta-analyses in head and neck cancer. Curr Opin Oncol 2007;19:188-94.

7 Pignon J-P, le Maître A, Maillard E, et al. Meta-analysis of chemotherapy in head and neck cancer (MACH-NC): an update on 93 randomised trials and 17,346 patients. Radiother Oncol 2009;92:4-14.

8 Vermorken JB, Remenar E, van Herpen C, et al. Cisplatin, fluorouracil, and docetaxel in unresectable head and neck cancer. N Engl J Med 2007;357:1695-704.

9 Haddad R, O'Neill A, Rabinowits G, et al. Induction chemotherapy followed by concurrent chemoradiotherapy (sequential chemoradiotherapy) versus concurrent chemoradiotherapy alone in locally advanced head and neck cancer (PARADIGM): a randomised phase 3 trial. Lancet Oncol 2013;14:257-64.

10 Abernethy AP, Etheredge LM, Ganz P, et al. Rapid-learning system for cancer care. J Clin Oncol 2010;28:4268-74.

11 Powell C, Schmidt M, Borri M, et al. Changes in functional imaging parameters following induction chemotherapy have important implications for individualised patient-based treatment regimens for advanced head and neck cancer. Radiother Oncol 2013;106:112-7.

12 Strongin A, Yovino S, Taylor R, et al. Primary tumor volume is an important predictor of clinical outcomes among patients with locally advanced squamous cell cancer of the head and neck treated with definitive chemoradiotherapy. Int J Radiat Oncol Biol Phys 2012;82:1823-30.

13 Grégoire V, Eisbruch A, Hamoir M, et al. Proposal for the delineation of the nodal CTV in the node-positive and the post-operative neck. Radiother Oncol 2006;79:15-20.

14 Harrell FEJ. Resampling,validating, describing, and simplifying the model. In: Harrell FEJ (Ed.). Regression Modeling Strategies, New York: Springer-Verlag 2001, pp. 87-103. 
15 Prestwich RJD, Sykes J, Carey B, et al. Improving target definition for head and neck radiotherapy: a place for magnetic resonance imaging and 18-fluoride fluorodeoxyglucose positron emission tomography? Clin Oncol (R Coll Radiol) 2012;24:577-89.

16 Hong TS, Tomé W, Harari PM. Heterogeneity in head and neck IMRT target design and clinical practice. Radiother Oncol 2012;103:92-8.

17 Njeh CF. Tumor delineation: The weakest link in the search for accuracy in radiotherapy. J Med Phys 2008;33:136-40.

18 Steenbakkers RJHM, Duppen JC, Fitton I, et al. Reduction of observer variation using matched CT-PET for lung cancer delineation: a three-dimensional analysis. Int J Radiat Oncol Biol Phys 2006;64:435-48.

19 Bayne M, Hicks RJ, Everitt S, et al. Reproducibility of "intelligent" contouring of gross tumor volume in non-small-cell lung cancer on PET/CT images using a standardized visual method. Int J Radiat Oncol Biol Phys 2010;77:1151-7.

20 Dimopoulos JC, De Vos V, Berger D, et al. Inter-observer comparison of target delineation for MRI-assisted cervical cancer brachytherapy: application of the GYN GEC-ESTRO recommendations. Radiother Oncol 2009;91:166-72.

21 Altorjai G, Fotina I, Lütgendorf-Caucig C, et al. Cone-beam CT-based delineation of stereotactic lung targets: the influence of image modality and target size on interobserver variability. Int J Radiat Oncol Biol Phys 2012;82:e265-72.

22 Cheebsumon P, van Velden FHP, Yaqub M, et al. Effects of image characteristics on performance of tumor delineation methods: a test-retest assessment. J Nucl Med 2011;52:1550-8.

23 Louie AV, Rodrigues G, Olsthoorn J, et al. Inter-observer and intra-observer reliability for lung cancer target volume delineation in the 4D-CT era. Radiother Oncol 2010;95:166-71.

${ }^{24}$ Fotina I, Lütgendorf-Caucig C, Stock M, et al. Critical discussion of evaluation parameters for inter-observer variability in target definition for radiation therapy. Strahlenther Onkol 2012;188:160-7.

25 Gevertz JL. Computational modeling of tumor response to vascular-targeting therapies - part I: validation. Comput Math Methods Med 2011;2011:1-17.

26 Araujo RP, McElwain DLS. A history of the study of solid tumour growth: the contribution of mathematical modelling. Bull Math Biol 2004;66:1039-91.

27 Joiner MC. Quantifying cell kill and cell survival. In: Joiner MC, van der Kogel AJ (Eds.). Basic Clinical Radiobiology, Fourth Ed. London: Hodder Arnold 2009, pp. 41-55.

28 Steyerberg EW, Vickers AJ, Cook NR, et al. Assessing the performance of prediction models: a framework for traditional and novel measures. Epidemiology 2010;21:128-38.

29 Machin D, Cheung YB, Parmar MKB. Survival Analysis. A practical approach. Second Ed. Chichester. England: John Wiley \& Sons 2006.
30 Iasonos A, Schrag D, Raj GV, et al. How to build and interpret a nomogram for cancer prognosis. J Clin Oncol 2008;26:1364-70.

31 Lütgendorf-Caucig C, Fotina I, Gallop-Evans E, et al. Multicenter evaluation of different target volume delineation concepts in pediatric Hodgkin's lymphoma. A case study. Strahlenther Onkol 2012;188:1025-30.

32 Bhatnagar P, Subesinghe M, Patel C, et al. Functional imaging for radiation treatment planning, response assessment, and adaptive therapy in head and neck cancer. Radiographics. 2013;33:1909-29.

33 Martínez Barbero JP, Rodríquez Jiménez I, Martin Noguer$\mathrm{ol} \mathrm{T}$, et al. Utility of MRI diffusion techniques in the evaluation of tumors of the head and neck. Cancers (Basel) 2013;5:875-89.

34 Bains LJ, Zweifel M, Thoeny HC. Therapy response with diffusion MRI: an update. Cancer Imaging 2012;12:395-402.

35 Noij DP, de Jong MC, Mulders LG, et al. Contrast-enhanced perfusion magnetic resonance imaging for head and neck squamous cell carcinoma: a systematic review. Oral Oncol 2015;51:124-38.

36 Shukla-Dave A, Lee NY, Jansen JF, et al. Dynamic contrastenhanced magnetic resonance imaging as a predictor of outcome in head-and-neck squamous cell carcinoma patients with nodal metastases. Int J Radiat Oncol Biol Phys 2012;82:1837-44.

37 Razek AA, Tawfik AM, Elsorogy LG, et al. Perfusion CT of head and neck cancer. Eur J Radiol 2014;83:537-44.

38 Popovic KS, Lukic S, Popovic P. Pretreatment perfusion CT and $C T$ volumetry in squamous cell carcinoma of the head and neck region. J BUON 2014;19:937-43.

39 Harrell FEJ. Multivariable Modeling Strategies. In: Harrell FEJ (Ed.). Regression Modeling Strategies. New York: Springer-Verlag 2001, pp. 53-85.

40 Cox DR. Regression Models and Life-Tables. J Royal Statistic Soc Series B (Methodological) 1972;34:187-220.

${ }^{41}$ Chu S-T, Wu P-H, Hou Y-Y, et al. Primary tumor volume of nasopharyngeal carcinoma: significance for recurrence and survival. J Chin Med Assoc 2008;71:461-6.

${ }^{42}$ Chua DT, Sham JS, Kwong DL, et al. Volumetric analysis of tumor extent in nasopharyngeal carcinoma and correlation with treatment outcome. Int J Radiat Oncol Biol Phys 1997;39:711-9.

43 Johnson CR, Khandelwal SR, Schmidt-Ullrich RK, et al. The influence of quantitative tumor volume measurements on local control in advanced head and neck cancer using concomitant boost accelerated superfractionated irradiation. Int J Radiat Oncol Biol Phys 1995;32:635-41.

${ }^{44}$ Lim K, Chan P, Dinniwell R, et al. Cervical cancer regression measured using weekly magnetic resonance imaging during fractionated radiotherapy: radiobiologic modeling and correlation with tumor hypoxia. Int J Radiat Oncol Biol Phys 2008;70:126-33. 